\title{
Multiscale method for characterization of porous microstructures and their impact on macroscopic effective permeability
}

\author{
W. C. $\operatorname{Sun}^{1}$, J. E. Andrade ${ }^{1, *, \dagger}$ and J. W. Rudnicki ${ }^{2}$ \\ ${ }^{1}$ Engineering and Applied Science, California Institute of Technology, Pasadena, CA 91125, U.S.A. \\ ${ }^{2}$ Department of Civil and Environmental Engineering, Northwestern University, Evanston, IL 60208, U.S.A.
}

\begin{abstract}
SUMMARY
Recent technology advancements on X-ray computed tomography (X-ray CT) offer a nondestructive approach to extract complex three-dimensional geometries with details as small as a few microns in size. This new technology opens the door to study the interplay between microscopic properties (e.g. porosity) and macroscopic fluid transport properties (e.g. permeability). To take full advantage of X-ray CT, we introduce a multiscale framework that relates macroscopic fluid transport behavior not only to porosity but also to other important microstructural attributes, such as occluded/connected porosity and geometrical tortuosity, which are extracted using new computational techniques from digital images of porous materials. In particular, we introduce level set methods, and concepts from graph theory, to determine the geometrical tortuosity and connected porosity, while using a lattice Boltzmann/finite element scheme to obtain homogenized effective permeability at specimen-scale. We showcase the applicability and efficiency of this multiscale framework by two examples, one using a synthetic array and another using a sample of natural sandstone with complex pore structure. Copyright (C) 2011 John Wiley \& Sons, Ltd.
\end{abstract}

Received 4 February 2011; Revised 4 April 2011; Accepted 7 April 2011

KEY WORDS: geometrical tortuosity; level set method; lattice Boltzmann/finite element method

\section{INTRODUCTION}

Understanding the interactions between the microstructural geometry and the macroscopic effective permeability of porous media is a constant challenge of longstanding interest to a variety of technological areas such as hydrology, chemical production processes, geological science and biomechanics. As a result, a vast number of theoretical and numerical studies have attempted to examine how pore-scale geometrical features of flow paths inside porous media influence the fluid diffusion phenomena observed at the macroscale. Many of these studies are oriented toward using capillary tube analogs or statistically reconstructed models to establish correlations or empirical relations between micro-structural attributes (e.g. grain diameters, grain size distribution, pore throat sizes, tortuosities, porosities) and macroscopic permeabilities. While these studies are useful, it is difficult to assess how well the analogs and statistically reconstructed models represent the actual three-dimensional pore geometry of real porous media. As a result, it remains unclear whether the findings based on analogs and reconstructed models are truly conclusive and applicable to porous media in general.

${ }^{*}$ Correspondence to: J. E. Andrade, Engineering and Applied Science, California Institute of Technology, Pasadena, CA 91125, U.S.A.

†E-mail: jandrade@caltech.edu 
A more desirable alternative is to directly calculate micro-structural attributes from the pore geometry reconstructed from three-dimensional tomographic images and relate them quantitatively to macroscopic variables. This is not a trivial task. Owing to the complexity of the pore geometry in real porous media, the calculations of effective permeabilities often require large-scale hydrodynamics simulations, which could be prohibitively expensive. Meanwhile, extracting pore geometry measurements, such as tortuosity and connected/occluded pore space, is very difficult without the prior knowledge of a geometrical object called the medial axis [1]. The construction of medial axes, however, is itself another computationally demanding task that requires special numerical treatment to handle complex geometries.

To overcome these challenges, we introduce a new computational framework that can rapidly extract the micro-structural attributes (e.g. geometrical tortuosity, connected/occluded porosity) and the macroscopic permeabilities in a systematic and computationally affordable manner. The major advantage of this computational framework is its ability to connect different numerical techniques sequentially and re-use outputs of one technique as input for the other ones in order to speed up the calculation and improve the accuracy. In particular, we introduce a new semi-implicit level set scheme to directly extract the three-dimensional medial axes that represent the geometry of the pore space. Using these 3D medial axes as starting point, we construct a mathematical object called weighted graph or weighted network to characterize the connectivity of the 3D medial axes. The weighted graph is used to determine shortest flow path via Dijkstra's algorithm [2]. These shortest flow paths in return give us the geometrical tortuosity. Then, by using the shortest flow path as input, we identify the connected/occluded pore space via a recursive procedure and use the knowledge of the connected pore space to speed up and improve the accuracy of the multiscale permeability calculations. In the multiscale permeability calculation, we calculate the macroscopic effective permeability via two homogenization procedures, one for micro-to-meso upscaling and one for meso-to-macro upscaling. To assess the accuracy of these homogenization procedures, we introduce a new parameter to quantitatively measure how close the multiscale model satisfies the Hill-Mandel condition [3,4].

The organization of this paper is as follows. First, we explain the big picture of the computational framework and how various techniques are put together to extract fluid properties and pore geometry measurements. Then, we discuss in more detail how geometrical tortuosities and connected/occluded pore space are determined. Following the pore-scale geometrical analysis, we present the multiscale framework to calculate macroscopic effective permeability at a scale relevant to engineering applications. The computational framework is tested by two examples in the application section, followed by the conclusion of this paper.

\section{OVERALL ARCHITECTURE}

As depicted in Figure 1, the methodology presented in this paper can be divided into two parts. Part 1 is furnished by a detailed geometrical analysis where two micromechanical attributes are extracted: geometrical tortuosity and connected porosity; both key factors are influencing macroscopic permeability. Part 2 of the method deals with effectively using the physical attributes extracted during part 1 and two-scale lattice Boltzmann (LB)/Finite element procedure to compute the macroscopic effective permeability of porous materials.

The central part of the method is part 1, where three numerical techniques are used to extract the geometrical tortuosity and connected porosity. First, the level set method is used to obtain the medial axes of the pore space. Second, a shortest path algorithm is used to extract the geometrical tortuosity. Third, a region-growing algorithm is used to identify the connected porosity, and by default the occluded porosity. It must be noted that these processes are performed sequentially, with the output from one serving as the input of the other. Armed with the micromechanical information obtained in part 1, we launch a two-scale homogenization procedure in which LB is implemented in the connected porosity only and the finite element method is then used to take the computations to the macroscopic scale. Domain decomposition and focusing the LB procedure on the connected 


\section{FLOWCHART OF THE MULTISCALE FRAMEWORK FOR PORE-FLUID TRANSPORT PROBLEM}

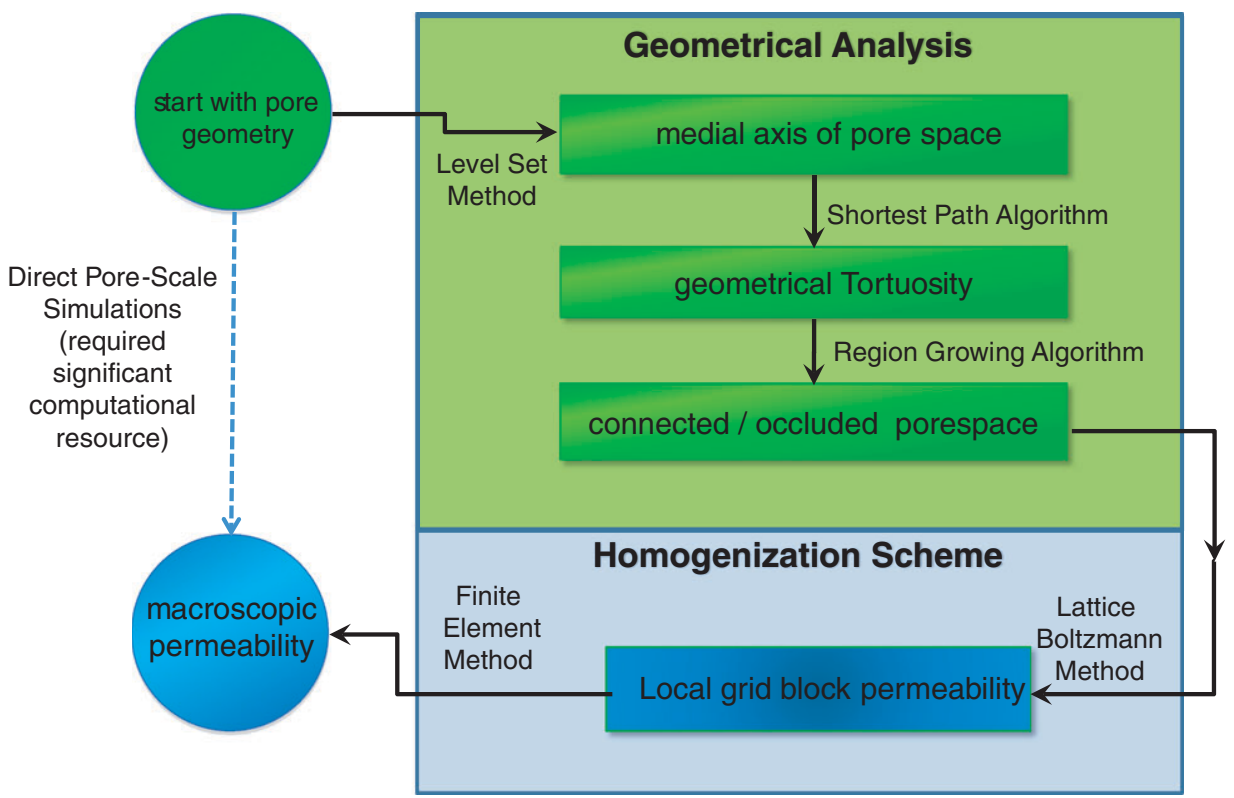

Figure 1. Flow chart of the numerical procedures used to compute tortuosity, identify connected/occluded pore space and estimate effective permeability at the specimen-scale.

porosity differentiates this method from its predecessors and makes it very efficient. In the next sections we describe the implementation of the main parts of the method in further detail.

\section{PART 1: NUMERICAL PROCEDURES FOR PORE-SCALE GEOMETRICAL ANALYSIS}

Our point of departure is the assumption that a digital representation of the porosity of the material is available. For example, a binary image of the solid phase and porous phase can be furnished using $\mathrm{X}$-ray CT. Once this microstructure is available, the question is how to use it to make quantitative statements of macroscopic properties such as permeability. To this end, we develop a numerical technique that relies on three interconnected components: development of level sets to determine the three-dimensional medial axes of the pore structure; development of shortest-path algorithm to evaluate the geometric tortuosity; and finally development of a growing-region algorithm to evaluate connected pore space. The medial axes can be seen as the spinal cord of the pore structure and the methodology presented herein.

\subsection{Semi-implicit variational level sets for medial axes extraction}

Medial axes are spine lines that trace the geometrical centers of volume-filling objects, such as flow channels formed by voids in a porous medium. Graphically, medial axes can be thought of as skeletons that inherit the shape of their corresponding volume-filling objects $[1,5]$. Thus, the length of the actual flow channels and that of the medial axes representing them are equal. This feature can be used to quantify geometrical properties of the pore space using well-known parameters such as geometrical tortuosity and connected porosity.

Typically, medial axes are obtained via thinning algorithms, such as the BURN algorithm used in $[1,5,6]$. These thinning algorithms share the same key idea: they remove the outer boundary layers of the volume-filling objects until these are thinned into curves. Here, we propose a new thinning algorithm based on the level set method. The main motivation for this new procedure is 


\section{Binary Image}

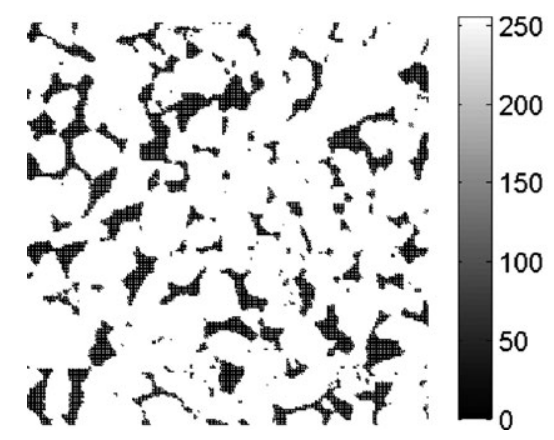

Edge Indicator

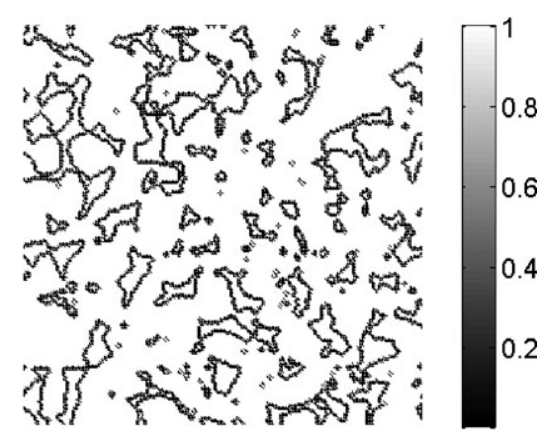

Figure 2. A binary image $\phi_{o}$ and its corresponding edge indicator function $g$.

computational efficiency as the level set method lends itself nicely to capture complex geometries in three-dimensional space.

Let us define the binary images describing the microstructure of the porous material as the field $\phi_{o}$. This is a bi-variate field, taking values equal to 255 to describe the solid skeleton and 0 to describe pore spaces. The initial field can be easily interpolated using a continuous function and the solid-void interfaces, $\Gamma$ can be determined by computing the gradient of $\phi_{o}$. Here, we define an edge indicator function $g$ such that

$$
g=\frac{1}{1+\nabla^{x} \phi_{o} \cdot \nabla^{x} \phi_{o}}
$$

where we have made use of the gradient operator $\nabla^{x}$. As shown in Figure 2, the numerical values of the edge indicator will be close to zero along the solid-void interfaces and equal to one elsewhere. Once the solid-void interfaces have been identified, we can use the level set method to determine the signed distance function.

A signed distance function $\phi(x)$ measures the signed shortest distance between the position $\boldsymbol{x}$ and the interface $\Gamma$, i.e.

$$
\phi(x)= \begin{cases}-\inf _{\boldsymbol{y} \in \Gamma}\|\boldsymbol{x}-\boldsymbol{y}\| & \text { if } \phi_{o}(\boldsymbol{x})=255 \\ +\inf _{\boldsymbol{y} \in \Gamma}\|\boldsymbol{x}-\boldsymbol{y}\| & \text { if } \phi_{o}(\boldsymbol{x})=0\end{cases}
$$

where inf denotes the infimum of a function. According to Equation (2), $\phi(x)$ is negative if $\boldsymbol{x}$ is inside the solid phase, positive if $\boldsymbol{x}$ is inside the pore phase, and equal to zero if $\boldsymbol{x} \in \Gamma$. For example, if we have a two-dimensional pore space resembling a circle, the corresponding signed distance would be a three-dimensional cone with the maximum value of $\phi$ at the center of the circle and $\phi=0$ on the boundary of the circle. Note that since $\phi(x)$ is a metric measuring the distance between position $\boldsymbol{x}$ and its closest point on the boundary $\boldsymbol{y} \in \Gamma, \phi(\boldsymbol{x})$ reaches its local maximum if $\boldsymbol{x}$ is located in the medial axis of the volume, i.e.

$$
\phi(\boldsymbol{x})=\inf _{\boldsymbol{y}_{1} \in \Gamma}\|\boldsymbol{x}-\boldsymbol{y}\|=\inf _{\boldsymbol{y}_{2} \in \Gamma}\|\boldsymbol{x}-\boldsymbol{y}\|
$$

where $\left\|\nabla^{x} \phi(x)\right\|=1$. Therefore, as shown in Figure 3, the medial axes of the pore space can be interpolated from the local maxima of the signed distance.

3.1.1. Variational formulation. A signed distance function corresponding to the pore space can be determined by a variety of level set evolution equations. A level set evolution equation is a specific type of PDE called Hamilton-Jacobi, which takes the form,

$$
\frac{\partial \phi}{\partial t}+H\left(\nabla^{x} \phi\right)=0
$$



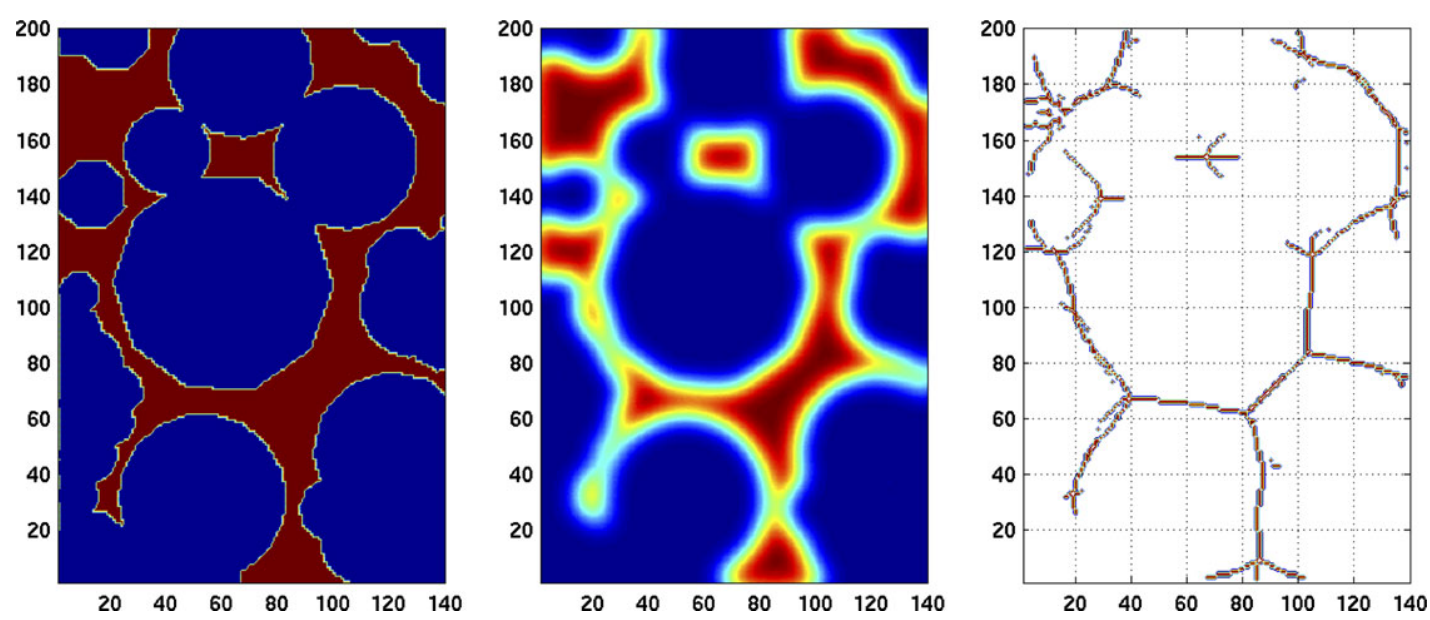

Figure 3. Two-dimensional pore space example. Left figure is the original binary image, containing only binary data. By applying the level set scheme, the signed distance function is formed inside the pore space as illustrated in the middle figure. The medial axis can be located by interpolating the local maximum point of the signed distance function as illustrated in the right figure.
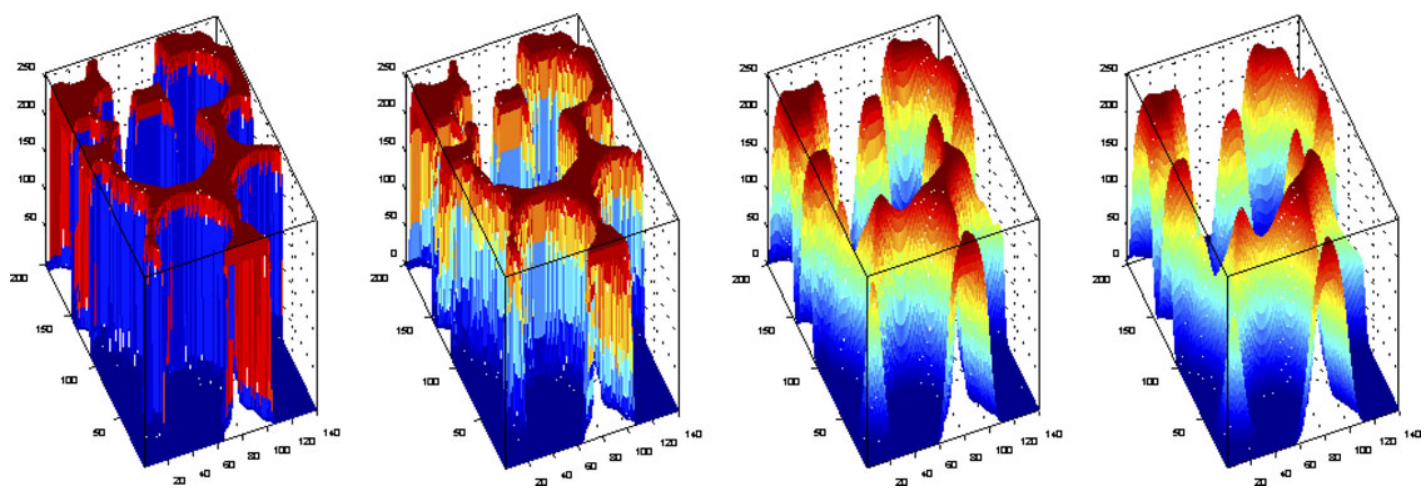

Figure 4. Example evolution of level set function $\phi$. The level set function converted into a signed distance function once reaching steady state of (9).

where $H$ is called the Hamiltonian. With a properly defined Hamiltonian, the Hamilton-Jacobi equation can force an arbitrary continuous function to evolve into a signed distance function as a function of time and as illustrated in Figure 4.

A particular form for the Hamiltonian has been proposed by Li et al. [7]. This formulation relies on a variational form and circumvents many of the problems associated with traditional level set evolution equations, in particular, re-initialization. An energy functional is introduced to enforce Equation (4) whereas other energy functionals are introduced to move the zero level set $\phi=0$ toward the solid-void interface. Herein, we adopt the formulation of Li et al. and implement it using a new numerical technique. The analytical formulation is briefly summarized below for completeness but further details can be found in [7].

An energy functional $\mathscr{E}$ is introduced such that $H=\partial \mathscr{E} / \partial \phi$, where the right-hand side is defined as the Gateaux derivative of the functional $\mathscr{E}$. The energy is defined as a linear combination of three fictitious energy terms such that

$$
\mathscr{E}(\phi)=\mu \mathscr{P}(\phi)+\lambda \mathscr{L}_{g}+v \mathscr{A}_{g}
$$


where $\mu, \lambda$, and $v$ are numerical parameters introduced to control the diffusion rate of the level set function. The energy (internal) $\mathscr{P}$ is a penalty functional to drive the level set function to satisfy $\left\|\nabla^{x} \phi\right\|=1$ such that,

$$
\mathscr{P}(\phi)=\int_{\Omega} \frac{1}{2}\left(\left\|\nabla^{x} \phi\right\|-1\right)^{2} \mathrm{~d} \Omega
$$

By the same token, the energies (external) $\mathscr{L}_{g}$ and $\mathscr{A}_{g}$ drive $\phi=0$ along solid-void boundaries. They can be expressed as

$$
\begin{aligned}
& \mathscr{L}_{g}(\phi)=\int_{\Omega} g \delta(\phi)\left\|\nabla^{x} \phi\right\| \mathrm{d} \Omega \\
& \mathscr{A}_{g}(\phi)=\int_{\Omega} g \hat{H}(-\phi) \mathrm{d} \Omega
\end{aligned}
$$

where $\delta$ is the univariate Dirac delta function and $\hat{H}$ is the Heaviside function. We note the dependence of $\mathscr{L}_{g}$ and $\mathscr{A}_{g}$ on the edge indicator function $g$. In addition, these latter functionals do not affect the value of $\phi$ along the solid-void boundaries, but are rather responsible for shrinking the level set function elsewhere such that the local maxima points of the level function coincide with those of the signed distance function. The governing equation (4) is then obtained by the principle of least action, where $\partial \mathscr{E} / \partial \phi=0$ at steady-state [7], and hence,

$$
\frac{\partial \phi}{\partial t}=\mu\left[\Delta^{x} \phi-\nabla^{x} \cdot \hat{\boldsymbol{n}}\right]+\lambda \delta(\phi) \nabla^{x} \cdot(g \hat{\boldsymbol{n}})+v g \delta(\phi)
$$

where $\Delta^{x}$ is the Laplacian operator and $\hat{\boldsymbol{n}}:=\nabla^{x} \phi /\left\|\nabla^{x} \phi\right\|$ and is a unit vector in the direction on the gradient of $\phi$. In the next section, we present a new algorithm to integrate the above equation.

3.1.2. Semi-implicit integration of variational formulation. In order to obtain a signed distance function for the pore space, it is necessary to integrate Equation (9) in space and time. In this work, we propose a semi-implicit scheme that can deliver stable, yet economical numerical solutions. Fully explicit solutions become problematic as they require a small time step to conserve stability. In the case of complex pore geometries, such as the ones encountered in natural geologic deposits, a small time step becomes a major handicap to the method. On the other hand, nonlinearities present on the Hamiltonian term (cf., right-hand side of Equation (9)) make fully implicit procedures difficult and expensive since they would require iterations. A good compromise seems to discretize the Hamiltonian semi-implicitly in time, with the Laplacian operator described fully implicitly. This is in contrast to the procedure presented in [7], where the Hamiltonian is integrated fully explicitly.

Integrating the governing equation in time using a forward difference scheme, we obtain

$$
\phi^{n+1}=\phi^{n}-\Delta t \bar{H}
$$

where $\phi^{n+1}$ corresponds to the $\phi$ field evaluated at the discrete time station $t=t_{n+1}$ and $\Delta t=$ $t_{n+1}-t_{n}$. In addition, for a given point in space, we can express the discrete Hamiltonian semiimplicitly by integrating in space using central differencing such that

$$
\bar{H}=-\mu\left[\Delta^{c} \phi^{n+1}-\nabla^{c} \cdot \hat{\boldsymbol{n}}^{n}\right]-\lambda \delta\left(\phi^{n}\right) \nabla^{c} \cdot\left(g \hat{\boldsymbol{n}}^{n}\right)-v g \delta\left(\phi^{n}\right)
$$

where we have used the discrete central difference operators for the gradient $\nabla^{c}$, the divergence $\nabla^{c}$. and the Laplacian $\Delta^{c}$, and $\hat{\boldsymbol{n}}^{n}=\nabla^{c} \phi^{n} /\left\|\nabla^{c} \phi^{n}\right\|$. We should emphasize that only the first term of the Hamiltonian, corresponding to the Laplacian operator, is expressed implicitly. Consequently, the current semi-implicit scheme does not require iterations, since the nonlinear terms in the Hamiltonian are treated explicitly (evaluated at time $t_{n}$ ). We implement this semi-implicit technique in the numerical examples presented herein and showcase the achieved numerical efficiency of the linear system and the stability afforded by an implicit technique. 


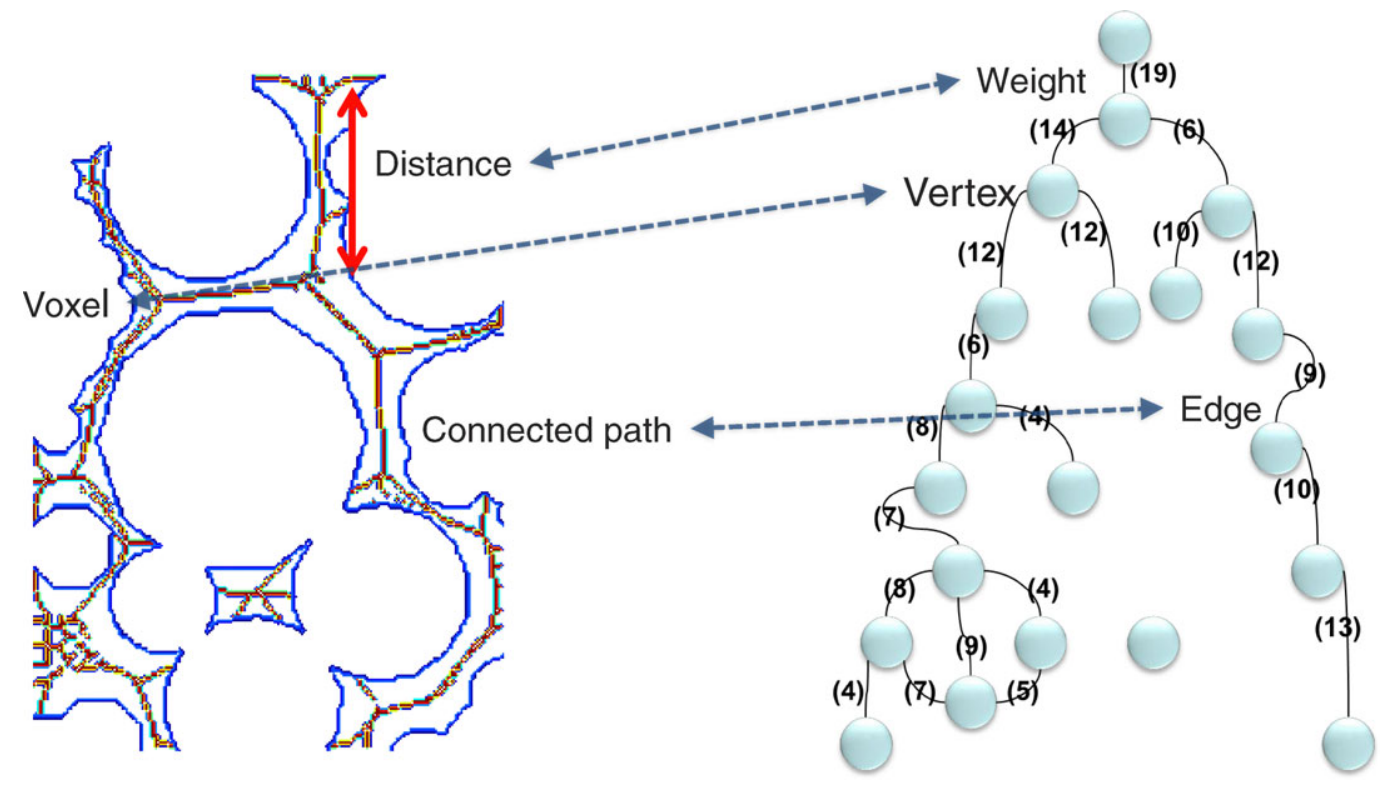

Figure 5. Example of corresponding weighted graph for sample medial axis. Medial axis corresponds to example shown in Figure 3.

\subsection{Dijkstra's algorithm for geometrical tortuosity extraction}

Fluid flow in porous media follows complex paths and it is directed by micro-channels. One parameter that attempts to quantify the complexity of these flow channels is the geometrical tortuosity $\tau$, defined as the ratio between the effective length of the shortest flow path $L_{\mathrm{e}}$ and the thickness of the porous medium $L[1,8,9]$, i.e.

$$
\tau=\frac{L_{\mathrm{e}}}{L}
$$

Everything else being equal, porous media with higher geometrical tortuosities require higher pore pressure gradients to achieve the same filtration rates, due to the effective elongation of the flow channels. For example, the widely used Kozeny-Carman equation [10] predicts that the effective permeability of a porous system is proportional to the reciprocal of tortuosity $1 / \tau$. Because of its important role in the flow properties of porous media, it is often desirable to measure the geometric tortuosity. One approach used by Lindquist and co-workers [1] is to apply a thinning algorithm on the inlet and outlet faces of the porous medium, then apply a shortest path searching algorithm on the entire pore space to determine the effective length. The major drawback of this approach is that it requires significant CPU time and memory usage due to the large number of voxels used to represent the $3 \mathrm{D}$ pore space.

In this work, we propose an alternative approach to measure the geometrical tortuosity. We apply the shortest path algorithm on the three-dimensional medial axis, instead of the entire pore space, obtaining significant savings on computing time. The procedure has as point of departure the medial axis information of the porous medium, as described previously. With the medial axis as input, we seek for the shortest path connecting opposite edges in a given volume. A key element of the algorithm is the definition of a weighted graph. A weighted graph is a network of nodes connected in between by a unique edge, and each edge has a weight [11]. As illustrated in Figure 5, in our case, each voxel in the medial axes is defined as a node, connected by edges whose weight is given by the Euclidean distance connecting the voxels. The objective of the graph is to provide a three-dimensional representation of the network and its connectivity. This will prove crucial for finding the shortest path. 
Armed with the weighted graph representing the medial axis of the porous medium, Dijkstra's algorithm [2] is used to determine the effective length and geometrical tortuosities of the graph (which correspond to those of the actual porous medium). In our particular problem, Dijkstra's algorithm works as follows. (1) Locate vertices that are on the inflow and outflow faces of the specimen (volume). (2) Label one of the vertices on the inflow face as the first active vertex and one of the vertices located on the outflow face as the targeted outflow vertex. (3) Use graph connectivity to select unvisited vertices directly connected to the active vertices and that are also connected to the outlet vertex. Selected vertices become part of the active set and the total length of paths defined by the active vertices are computed using the weights. Step (3) is repeated until either (i) the targeted outflow vertex becomes an active vertex or (ii) no unvisited vertex is next to an active vertex. In the case of (i), then the length of the shortest path connecting the inflow and outflow vertices is the effective length $L_{\mathrm{e}}$. Dijkstra's algorithm ranks all lengths of connecting flow channels by ascending order so the shortest path appears first when the algorithm reaches condition (i). If (ii) is reached without (i), the selected inflow and outflow vertices are not connected. We continue the procedure above until all possible pairs of inflow and outflow vertices have been examined. If there are $N_{1}$ inflow vertices and $N_{2}$ outflow vertices, the algorithm is run $N_{1} \times N_{2}$ times. In the example shown in Figure 5, the shortest path length is 69 and the algorithm is run twice.

\subsection{Region-growing algorithm for connected pore space extraction}

Previous research has focused on relating the effective permeability with the total porosity $[12,13]$. A major drawback of these approaches is that they do not distinguish the occluded porosity from its connected counterpart. Connected pore channels govern transport properties and, therefore, must be accurately evaluated. Fortunately, both occluded/connected porosities can be measured by using the information we have already acquired, i.e. the graph that represents the porous network and the shortest flow path extracted from the level set and Dijkstra's algorithms. Since the shortest flow path is inside the connected pore space, we can identify the connected pore space by examining the neighboring voxels and classifying them as connected pore space until reaching the boundaries.

To identify the connected/occluded pore space, we first use the flow path obtained from Dijkstra's algorithm as seeds planted inside the connected pore space. Then, a recursive function is used to simulate the growth of the spanning tree stemmed from the seeds. This recursive function stops running when all the edges inside the connected pore space are explored and all vertices inside the connected pore space are visited. Since the occluded and connected pore space are mutually exclusive, the region of pore space not visited by the recursive function is therefore the occluded pore space. The pseudo-code of the computer program used to identify connected/occluded pore space is as given in Box 1 .

BOX 1: Pseudocode of the program used to identify connected pore space.

1. Activate all vertices along the flow path as active nodes and mark them as visited vertices

2. While there exists at least one active node

(a) call the recursive function MARKNEIGHBOR

3. EXIT

\section{FUNCTION MARKNEIGHBOR}

1. IF at least one neighbor of the active nodes has not yet been visited

(a) Activate the unvisited neighbor vertices.

(b) Mark them as visited vertices.

(c) Deactivate the old active nodes with unvisited neighbor(s).

(d) Call the recursive function MARKNEIGHBOR.

2. ELSE

(a) Deactivate the active nodes with no unvisited neighbor.

3. EXIT 


\section{PART 2: TWO-SCALE HOMOGENIZATION OF PERMEABILITY USING LB AND FEM}

The effective permeability of a porous medium can be measured by applying a pore pressure gradient along a basis direction and determining the resultant fluid filtration velocity from porescale hydrodynamics simulation. Then the effective permeability tensor can be obtained according to Darcy's law

$$
k_{i j}=-\frac{\mu^{v}}{p, j}\left\langle v_{i}\right\rangle
$$

where $v_{i}$ is the flow vector in the $i$ th orthogonal direction, $\langle\square\rangle$ represents volume average, $\mu^{v}$ is the kinematic viscosity of the fluid, and $p,{ }_{j}$ is the gradient in fluid pressure in the $j$ th orthogonal direction. The effective permeability tensor $k_{i j}$ is treated here in the standard way, where it is assumed to be diagonal and positive definite. Hence, only the diagonal components of the tensor are non-zero and need to be evaluated.

One way to calculate the effective permeability in a porous sample is to perform a mesoscale direct numerical simulation (e.g. using LB) over the entire sample and in this way account for all details in pore geometry. This direct approach, though accurate, requires immense memory and CPU usage times for specimens of sizes relevant to engineering applications. A significantly cheaper way to calculate permeability at the specimen scale is to use a multiscale framework exploiting an LB/finite element hybrid scheme [13,14]. The key idea of the hybrid scheme is to apply domain decomposition and thereby break down the computationally demanding large simulation into multiple smaller problems that can be handled with less resources, then perform homogenization to obtain the equivalent macroscopic properties, as illustrated in Figure 6. We note here that while domain decomposition can reduce computational expenses significantly, it can potentially introduce considerable error if the occluded porosity is interpreted to be connected in the calculations. To avoid this issue, we perform all calculations using the connected porosity only as identified in the previous sections.

The two-scale domain decomposition scheme is illustrated in Figure 6. The scheme uses LB for the mesoscale calculations and finite elements for macroscopic simulations. First, the connected pore space in the sample is decomposed into smaller, manageable domains for direct mesoscale calculation (e.g. using LB). Permeability tensors are obtained for each subdomain using Equation (13) using LB on the connected porosity only. Second, each subdomain is represented geometrically by finite elements with permeabilities obtained from the previous step. Finally, finite elements

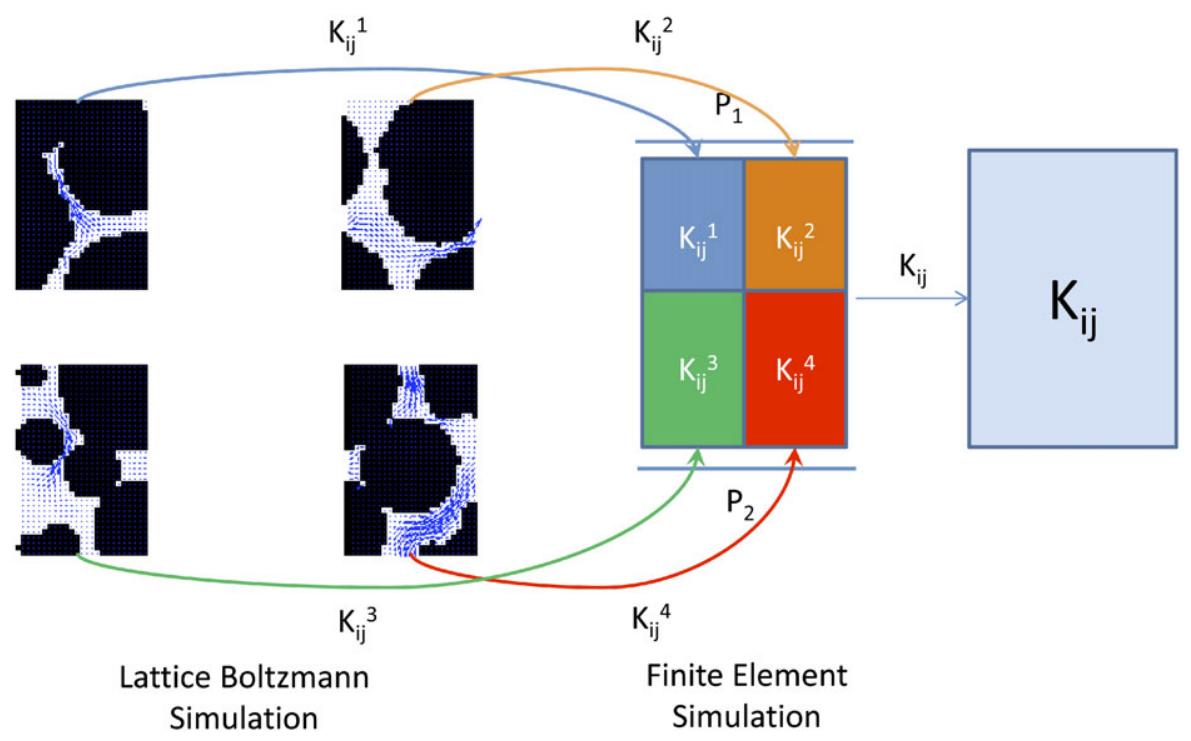

Figure 6. Multiscale numerical scheme used to determine effective permeability in large scale. 
(FEM) are used to estimate the effective permeability of the entire sample, accounting for the heterogeneities implied in each subdomain. This procedure is clearly shown in Figure 6 where the sample is decomposed into four subdomains. Permeability calculations are performed in each subdomain by LB and then these values are used in one more macroscale simulation using FEM to estimate the effective permeability of the entire sample.

\subsection{Numerical procedures: $L B$ and finite elements}

The main features of the LB and finite element procedures used in this work are summarized in this section for the sake of completion. In the LB procedure, the discrete distribution function $f_{i}(\boldsymbol{x}, t)$ is the main unknown such that the particle distribution satisfies the lattice Boltzmann equation [12-17], i.e.

$$
\frac{\partial f_{i}}{\partial t}+\boldsymbol{e}_{i} \cdot \nabla^{x} f_{i}=C_{i}
$$

where $C_{i}$ is a collision term that accounts for the net addition of particles moving with velocity $\boldsymbol{e}_{i}$ due to inter-particle collisions. LB is particularly suited to handle complex geometries such as those encountered in natural geomaterials. In addition, fluid velocity $v$ and pressure $p$ at lattice node $\boldsymbol{x}$ and time $t$ are both determined from the discrete distribution functions, i.e.

$$
\boldsymbol{v}=\frac{1}{\rho} \sum_{i=1}^{\alpha} f_{i} \boldsymbol{e}_{i}, \quad p=c^{2} \rho, \quad \rho=\sum_{i=1}^{\alpha} f_{i}
$$

where $\alpha$ is the number of lattice directions a molecule can move, and $c$ denotes the speed of sound, which is treated as a constant in the LB simulations. Using a simple standard technique proposed in $[16,18]$, we can reproduce nearly incompressible flows with velocities and pressures that can be used in Equation (13) to determine the mesoscale value of permeability.

After extracting the local permeability tensors for all unit cells, we assign the numerical values of these local permeability tensors to the corresponding Gauss points of the finite element model. The finite element model is aimed to simulate the macroscopic diffusion of an incompressible, singlephase pore-fluid. It is based on Darcy's law augmented with the incompressible constraint, i.e.

$$
\begin{aligned}
& \nabla^{x} \cdot v(x)=0 \\
& v(x)=-\frac{1}{\mu^{v}} k(x) \cdot \nabla^{x} p(x)
\end{aligned}
$$

where body forces are neglected and $\boldsymbol{k}=\boldsymbol{k}^{\text {meso }}$ denotes the local permeability tensor obtained from the mesoscale LB simulations described above. Combining Equations (16) and (17) yields a single-phase pressure equation for steady incompressible flow, i.e.

$$
\frac{1}{\mu^{v}} \nabla^{x} \cdot\left(\boldsymbol{k}(\boldsymbol{x}) \cdot \nabla^{\boldsymbol{x}} p(\boldsymbol{x})\right)=0
$$

Augmenting Equation (18) with the pressure prescribed on the corresponding boundaries, we obtain the boundary value problem suitable for finite element discretization. We use the standard Galerkin method to obtain the macroscopic pressure field.

\subsection{Upscaling effective permeability}

Accuracy and efficiency of the multiscale hybrid method rely crucially on the size selected for the unit cells. If the unit cells are too large, the speed of the multiscale method decreases dramatically; if the unit cells are too small, the multiscale method may fail, since the continuum representation may break down $[13,14]$. One way to strike some balance between accuracy and efficiency is to choose unit cells that are just big enough as to satisfy the continuum requirements. A unit cell that fits this description can be referred to as representative element volume (REV). While 
there is no universal rule to determine the appropriate size of REV, it is widely accepted that a REV must satisfy the following conditions [19]: (i) the REV must be large enough to contain sufficient statistical information about the microstructure; (ii) the effective constitutive response (e.g. permeability) must be independent of the type of boundary conditions imposed on the REV. The concept of REV, and conditions related to it, have been amply studied in the context of elasticity. Of particular importance is the condition known as the Hill-Mandel condition [3].

In this work, we will extend the Hill-Mandel condition for the application of flow through porous media. Our point of departure is the governing equation (18) and Dirichlet or Newmann uniform boundary conditions, analogous to the uniform stress and strain conditions in elasticity [4], i.e.

$$
\begin{gathered}
\boldsymbol{v} \cdot \boldsymbol{n}=\boldsymbol{v}_{0} \cdot \boldsymbol{n} \quad \text { on } \Gamma \quad \text { or } \\
p=p_{0} \quad \text { on } \Gamma
\end{gathered}
$$

where $\boldsymbol{n}$ is the outward normal to the boundary $\Gamma$ and $\boldsymbol{v}_{0}$ and $p_{0}$ are constant prescribed values of $v$ and $p$. Using the formulation in [20], one can show that (18) can be reformulated via the least action principle. In this case, the solution of Darcy's law augmented with continuity equation is the $p$ that minimizes the energy dissipation rate $D(p)$, i.e.

$$
\left.\frac{\partial D(p+\alpha \delta p)}{\partial \alpha}\right|_{\alpha=0}=0 \Leftrightarrow \frac{1}{\mu^{v}} \nabla^{x} \cdot\left(\boldsymbol{k} \cdot \nabla^{x} p\right)=0
$$

where the energy dissipation rate reads,

$$
D(p)=\frac{1}{\mu^{v}} \nabla^{x} p \cdot \boldsymbol{k} \cdot \nabla^{x} p
$$

The role of this energy dissipation rate for the pore-fluid transport problem is analogous to the elastic strain energy for the elasticity problem.

Consequently, the necessary condition for both (i) and (ii) for flow in heterogeneous porous media can be regarded as a special form of the Hill-Mandel condition [3, 4], which reads,

$$
D^{\text {macro }}=\frac{1}{\Omega} \int_{\Omega} \nabla^{x} p \cdot v \mathrm{~d} \Omega=\frac{1}{\Omega} \int_{\Omega} \nabla^{x} p \mathrm{~d} \Omega \cdot \frac{1}{\Omega} \int_{\Omega} v \mathrm{~d} \Omega
$$

where $D^{\text {macro }}$ is the macroscopic energy dissipation rate. By the divergence theorem, substituting Equation (17) into Equation (23), and assuming that the prescribed pressure is applied on the top and bottom of the numerical specimen, we obtain the following expression:

$$
D^{\text {macro }}=\frac{1}{\Omega} \int_{\Omega} \frac{1}{\mu^{v}} \nabla^{x} p \cdot \boldsymbol{k}^{\text {meso }} \cdot \nabla^{x} p \mathrm{~d} \Omega=\frac{k_{z z}^{\text {macro }}}{\mu^{v}} \frac{\left(p_{2}-p_{1}\right)^{2}}{\left(z_{2}-z_{1}\right)^{2}}
$$

where $\boldsymbol{k}^{\text {meso }}$ is the local permeability determined from the pore-scale LB simulations and $\boldsymbol{k}^{\text {macro }}$ is the global permeability obtained from the specimen-scale finite element simulations. In addition, $p_{2}$ and $p_{1}$ and $z_{2}$ and $z_{1}$ are the pressures and vertical positions of the outlet and inlet faces, respectively. Suffice it to say, Hill-Mandel's condition, as stated in Equation (24), is the criterion that ensures the energy dissipation rate calculated from the boundary values of the macroscopic pressure field is equal to the energy dissipation rate calculated from the volume integration over the numerical specimen. For an arbitrary porous medium, there may not always exist a definite mesoscale for which Hill-Mandel's condition holds exactly. Nevertheless, the difference between the macroscopic energy dissipation rate obtained from the boundary and volume integrations is still a good indicator that quantifies how accurately the homogenization scheme performs. In our calculations, we monitor the difference in these dissipations to ensure the accuracy of the upscaling from the local permeability tensor field $\boldsymbol{k}^{\text {meso }}$ to the global permeability tensor $\boldsymbol{k}^{\text {macro }}$.

In addition to proposing the use of $D^{\text {macro }}$ to ensure accuracy in going from meso to macroscale, we propose the use of energy dissipation $D^{\text {micro }}$ to ensure proper transition from micro- (from LB) to mesoscale. This approach was originally proposed by White et al. [14] in which the scale 


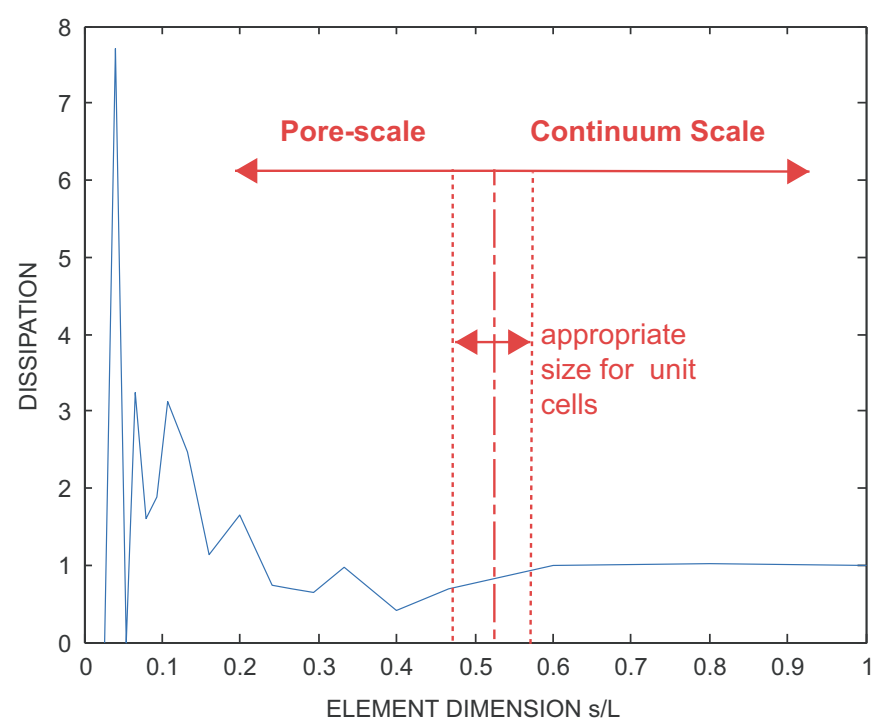

Figure 7. Selection of the unit cell size based on the scale of fluctuation.

fluctuation of the microscopic energy dissipation rate is used to determine the appropriate size of unit cell for the LB simulation. The microscopic energy dissipation rate is obtained from the LB simulation, such that,

$$
D^{\text {micro }}=2 \mu^{v} \epsilon: \epsilon ; \quad \epsilon=\frac{1}{2}\left(\nabla^{x} v+\left(\nabla^{x} v\right)^{\mathrm{T}}\right)
$$

where the local permeability tensor and the microscopic energy dissipation rate over the domain of the unit cell $\Omega^{c}$ satisfy the following relation:

$$
\frac{1}{\Omega^{c}} \int_{\Omega^{c}} D^{\text {micro }} \mathrm{d} \Omega^{c}=\nabla^{x} p \cdot \boldsymbol{k}^{\text {meso }} \cdot \nabla^{x} p
$$

To ensure that the LB simulations are conducted in a unit cell capable of resembling continuum behavior, we conduct a series of simulations on spatial domains with increasing size but fixed centroid. Then, we examine the scale of fluctuation of $D^{\text {micro }}$ versus the unit cell size and select the unit cell size that ameliorates the local fluctuation in energy dissipation, as illustrated in Figure 7.

\subsection{Remarks on occluded porosity and its impact on homogenized permeability}

In many situations, particularly in natural porous materials, occluded porosity occupies a significant portion of the pore space. Failure to identify that occluded porosity can cause dramatic errors in multiscale modes. Typical situations where significant occluded porosity is expected to play a role include the migration of pore-fill cement into pore space [21], pore closure in limestones due to $\mathrm{CO}_{2}$ sequestration [22], and the formation of compaction bands [23].

To illustrate this point, let us consider a two-dimensional LB simulation of the sample depicted in Figure 8. In this example, our objective is to obtain the vertical global permeability of a sample, discretized using a $30 u \times 40 u$ lattice ( $u=$ lattice unit), using three different techniques. In the first technique, LB simulations are conducted on the entire sample, without any domain decomposition. This is equivalent to a direct numerical simulation and is interpreted here as the 'true' solution. The second technique uses domain decomposition (sample is split into four parts along the vertical direction) and uses occluded space detection, keeping only connected porosity active. The third technique uses domain decomposition but does not distinguish between connected and occluded 


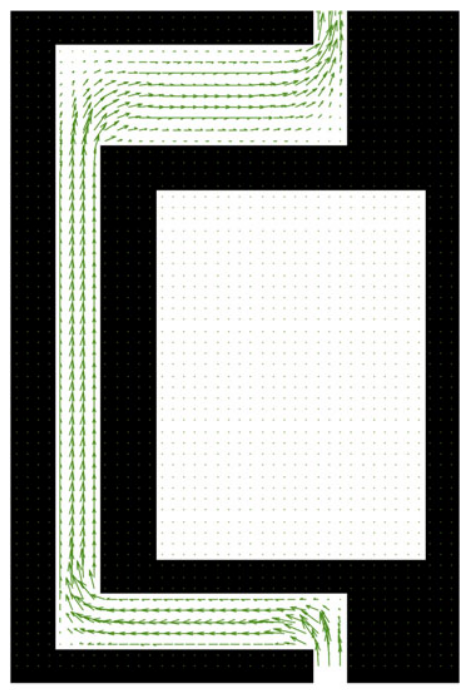

(a)
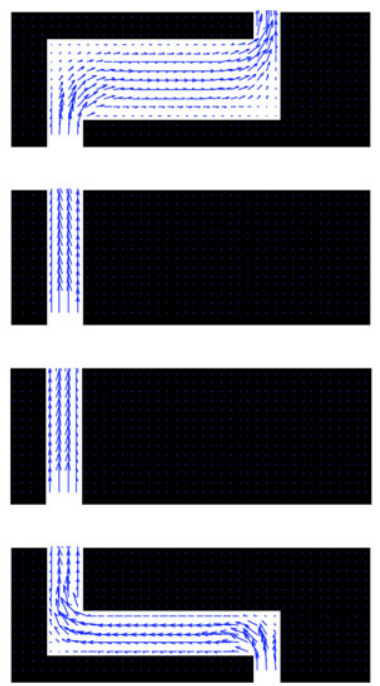

(b)
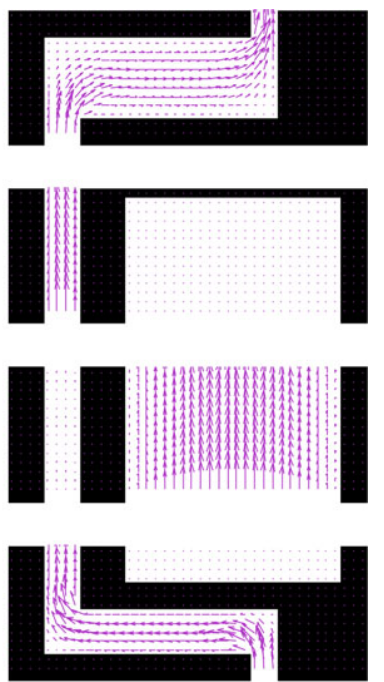

(c)

Figure 8. Velocity profiles of lattice Boltzmann simulations on: (a) unpartitioned domain $\left(k=0.015 u^{2}\right)$;

(b) partitioned domain with identified and deactivated occluded porosity $\left(k=0.013 u^{2}\right)$; and (c) partitioned domain without any special treatment for occluded porosity $\left(k=0.0078 u^{2}\right)$. Where $u=$ lattice unit.

Table I. Global and local permeabilities obtained from LB simulation scenarios.

\begin{tabular}{lccc}
\hline Case & 1 & 2 & 3 \\
\hline Number of Unit Cell(s) & 1 & 4 & 4 \\
Occluded Pore Identified? & No & Yes & No \\
Local Permeability, $u^{2}$ (top) & N/A & 0.011 & 0.011 \\
Local Permeability, $u^{2}$ (2nd top) & N/A & 0.015 & 0.0029 \\
Local Permeability, $u^{2}$ (2nd bottom) & N/A & 0.015 & 0.45 \\
Local Permeability, $u^{2}$ (bottom) & N/A & 0.014 & 0.014 \\
Global Permeability, $u^{2}$ & 0.015 & 0.013 & 0.0078 \\
Relative Error & 0 & $12 \%$ & $48 \%$ \\
\hline
\end{tabular}

porosity. Global permeabilities for the partitioned samples are obtained from the local estimates by [19],

$$
k=\frac{\sum_{i=1}^{n} L_{i}}{\sum_{i=1}^{n} L_{i} / k_{i}}
$$

where $k_{i}$ are the local values of permeability in each layer of thickness $L_{i}$, and $n=4$ denotes the number of unit cells (layers).

Results for the LB calculations are summarized in Table I. It should be highlighted that the relative error induced by the third procedure with partition but no special treatment of occluded porosity is four times greater than that of the partitioned method that takes into account occluded porosity. It can be seen from Table I that the main sources of error come from the mistreatment of occluded porosities in the central partitions. The mistreatment of occluded porosity is not only the source of errors in the estimation of permeability, but it leads to longer calculations as occluded porosity is assigned active lattices. Hence, not accounting for occluded porosity may lead to inaccuracies and inefficiencies. 


\section{REPRESENTATIVE EXAMPLES}

In this section, we present two example applications of the proposed framework. The first example deals with a spatially periodic, bi-continuous pore structure furnished by a simple cubic (SC) spherical array. The objective of this example is to verify the accuracy of the proposed method. Owing to the simplicity of the SC pore structure, there is a wealth of theoretical and numerical solutions in the literature that can be used to verify our newly proposed approach. The second example deals with a natural sample of Aztec sandstone inside a compaction band that has been imaged using synchrotron X-ray CT [12]. Compaction bands are a type of strain localization known to significantly reduce connected porosity and increase geometric tortuosity, thereby reducing the permeability of sandstones within these formations by orders of magnitude. Because of their possible importance for injection and extraction of fluids, compaction bands have been amply studied $[13,14,17,23-25]$. However, there are no direct measurements of permeability inside compaction bands from field samples in three dimensions. This example highlights the importance and applicability of the proposed framework in real porous media.

\subsection{Periodic simple cubic (SC) lattice}

Simple cubic (SC) cells can be formed by placing the centroid of eight identical spheres at the corners of a cube of equal dimensions. When the spheres are making point contact, it is often called SC bead pack [26]. In this packing, the total porosity is simply $\phi^{f}=1-\pi / 6$ and the geometrical tortuosity is simply unity, as the shortest flow path is one directly though the center of the cell. Furthermore, as in other simple packings, all porosity is connected.

Unlike micro-structural attributes, the permeability of SC packings cannot be directly obtained using analytical techniques. Instead, numerical procedures are often employed. The closest analytical solutions are furnished by bounds, such as the lower bound obtained by Dormieux and co-workers [9] where pore spaces are ordered in the sense of inclusions and the permeability of a cylinder with cross-section made up by four circles examined. Since the cylindrical pore space is a subset of that of the SC cell, the permeability of the cylindrical pore space serves as a lower bound for that of the SC cell. The lower bound can be expressed in dimensionless form as $k \geqslant 4.84 \times 10^{-3} R^{2}$, where $R$ is the radius of the spheres in the SC cell. Naturally, the permeability tensor in the SC cell is isotropic. Additionally, Zick and Homsy [27] have analyzed the permeability of the SC bead pack by reducing the Navier-Stokes equation to a set of Fredholm integral equations. They found $k=5.04 \times 10^{-3} R^{2}$.

Using the aforementioned studies as backdrop for the accuracy of our proposed method, we perform permeability calculations using the SC bead pack. Our first task is to correctly identify the connected porosity in the sample. The pore geometry is discretized in the usual way using a lattice mesh. The resolution of the lattice, clearly affects the results of the computations. The center of the pore space is selected as the first active lattice and porosity is determined using the region-growing algorithm described in Section 3.3. Figure 9 shows the estimate of porosity as a function of the lattice resolution. Once the voxel length is smaller that $R / 20$, the numerical solution closely captures the exact solution $(1-\pi / 6)$, with an error around $3 \%$.

To check whether the computational framework can determine the geometrical tortuosity, we apply the variational scheme and Dijkstra's algorithm as described in Section 3.2. Using a resolution of $R / 50$, the resultant level set function and the shortest flow path are illustrated in Figure 10. As shown in the figure, in this simple example the geometrical tortuosity is unity and Dijkstra's algorithm is able to obtain this result without any difficulty. Finally, turning our attention to the effective permeability calculation, we obtain an estimate using LB at the aforementioned lattice resolution. In addition, we carried out a three-dimensional Navier-Stokes finite element simulation to examine the reproducibility of the permeability calculation. The FE model is composed of 8937 tetrahedral Crouzeix-Raviat elements [28] with non-periodic side walls and prescribed pressures on the top and bottom faces of the cubic domain. The FE model was solved using an opensource differential solver called FEniCS [29]. Figure 11 illustrates the results of the LB and FE simulations. The permeability using LB and FE is estimated to be $4.64 \times 10^{-3} R^{2}$ and $4.89 \times$ 


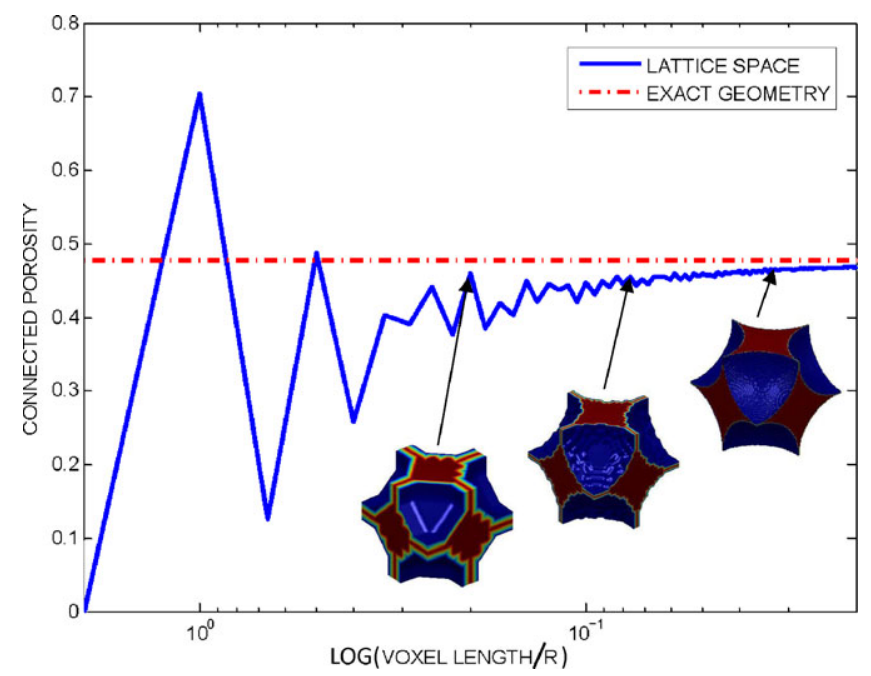

Figure 9. Connected porosity as a function of voxel length over radius in an SC bead packing.
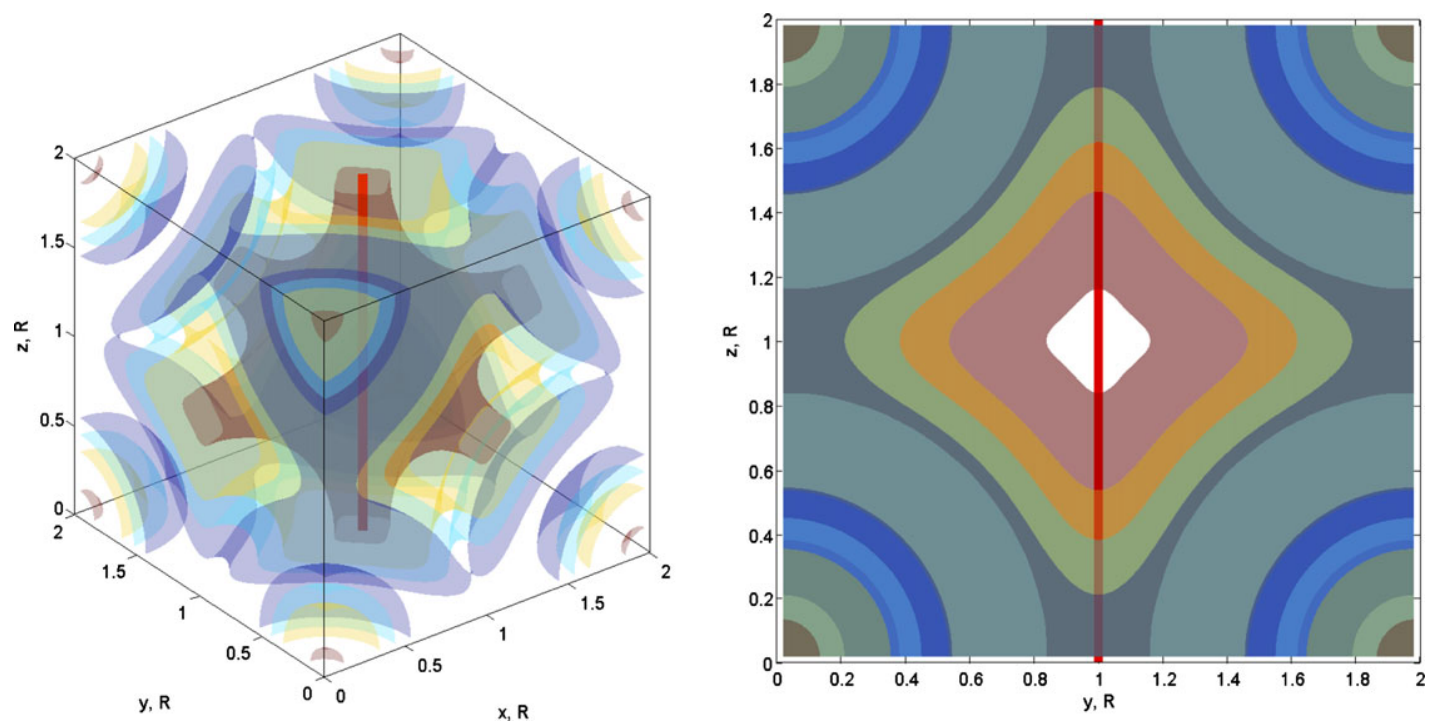

Figure 10. Level set function $\phi(x, y, z)$ (represented by the $3 \mathrm{D}$ color contour) and the corresponding shortest flow path (represented by the red straight line) as determined by Dijkstra's algorithm.

$10^{-3} R^{2}$, respectively. Since both methods are inherently different, and since the calculations are close to the previous values of permeability estimated for SC packings, we consider the $5.1 \%$ difference in solutions acceptable. We therefore conclude that the proposed framework to estimate permeability based on level sets and LB is accurate.

\subsection{Permeability calculation on natural complex porosity (e.g. compaction bands)}

In this section, we demonstrate the applicability of the proposed methodology to a complex porous network furnished by a natural sample of Aztec sandstone from the Valley of Fire, Nevada. A full tomographic image was obtained from a prismatic sample of $2.25 \times 2.25 \times 6.00 \mathrm{~mm}$ at a resolution of $6 \mu$. The sample is cored in the field from a naturally formed compaction band and the mean grain size is $0.25 \mathrm{~mm}$. The compaction band sample is studied here because it furnishes a very complex network of connected porosity, which has been significantly reduced from the natural rock 

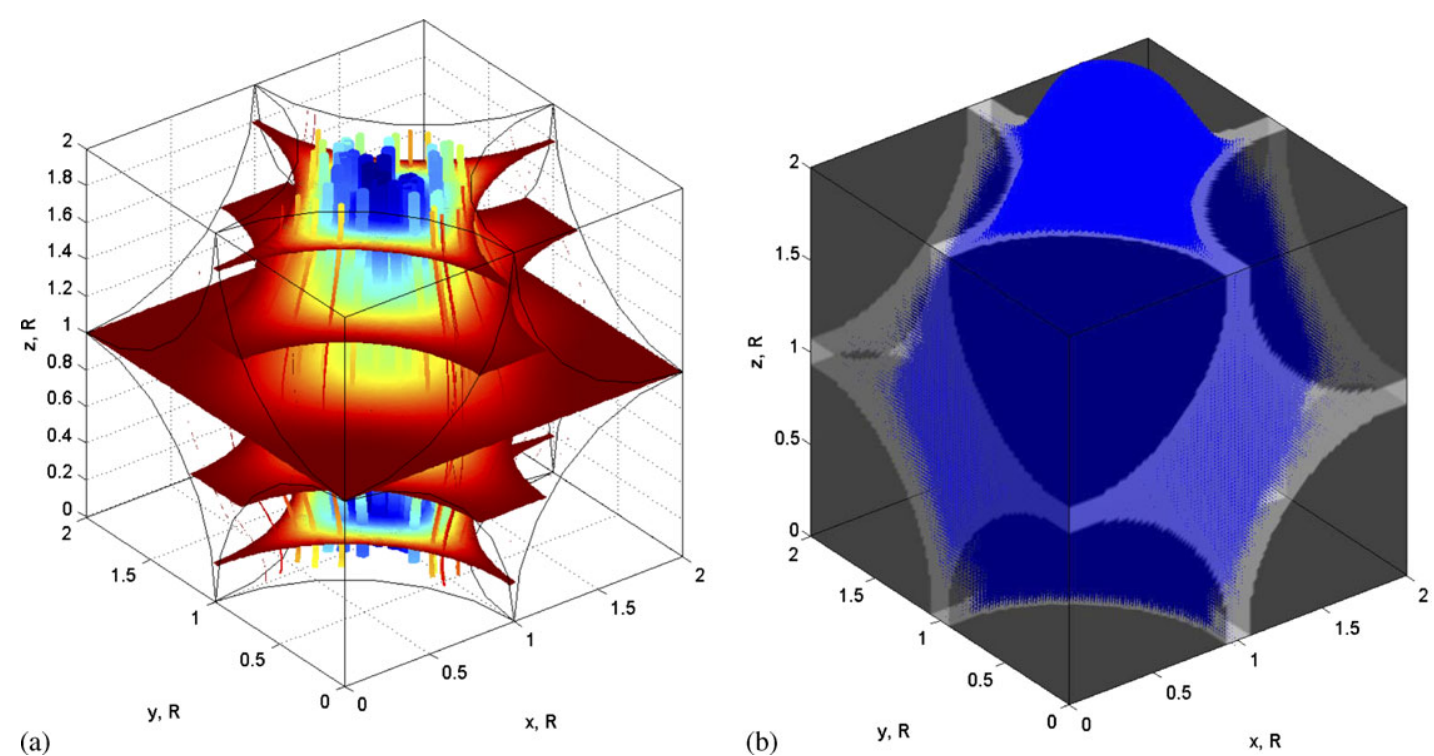

(a)

Figure 11. (a) Streamline of the simple cubic lattice computed via Stokes finite element model $\left(k=4.89 \times 10^{-3} R^{2}\right)$. Color map represents the magnitude of the velocity field. (b) Velocity profile of the simple cubic lattice obtained via lattice Boltzmann simulation conducted on connected pore space $\left(k=4.64 \times 10^{-3} R^{2}\right)$. The intensity of the blue color represents the magnitude of the fluid velocity field.
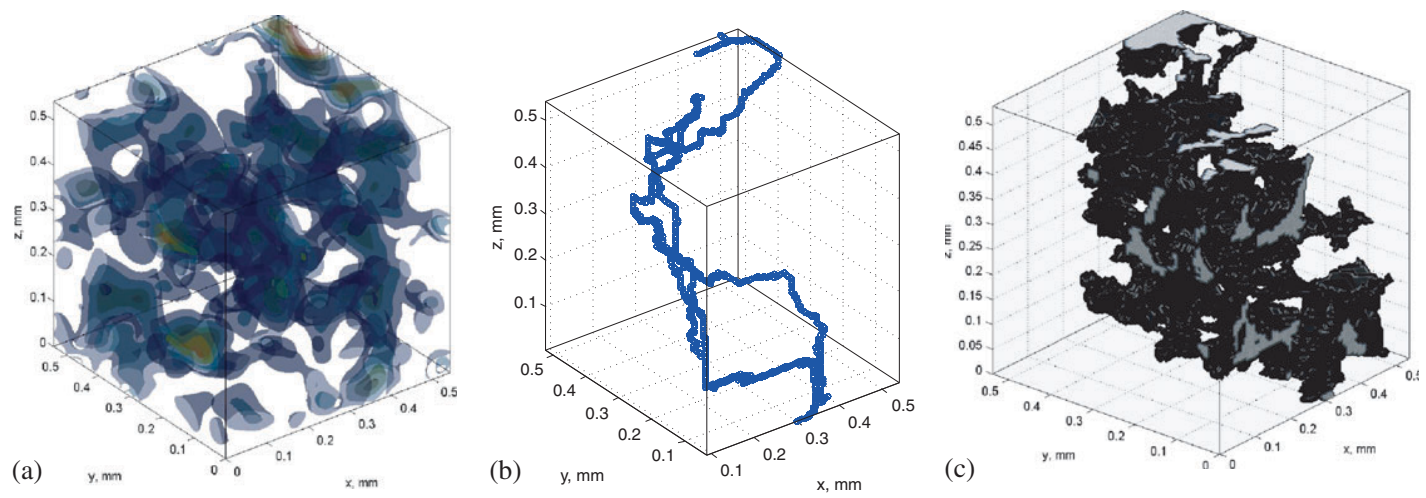

Figure 12. (a) Level set functions; (b) flow paths; and (c) connected pore space for compaction band cell with dimensions $0.54 \times 0.54 \times 0.54 \mathrm{~mm}$.

formation. The total porosity in the compaction band sample is $14 \%$, whereas the total porosity in the host rock is $21 \%$. More information about the sample and the geologic conditions can be found in [12].

Figure 12 shows a small cell of dimensions $0.54 \times 0.54 \times 0.54 \mathrm{~mm}$ along with the level set functions, connected flow channels, and connected porosity. We can see that the methodology presented above is able to extract a very complex network of porosity and can identify the connected porosity. In addition, Dijkstra's algorithm has been run to obtain the shortest flow paths shown in the figure. From these paths, the geometric tortuosity can be extracted, yielding an average tortuosity inside the compaction band of 2.79. This should be contrasted with the tortuosity in the host rock which is around 1.77. Hence, we can conclude that compaction bands increase the geometric tortuosity considerably.

Once the shortest flow paths are determined, we use the region-growing algorithm to obtain the connected porosity and differentiate this from the total porosity. As mentioned before, the regiongrowing algorithm uses voxels located on the shortest flow paths as seeds. Then, the connected pore 


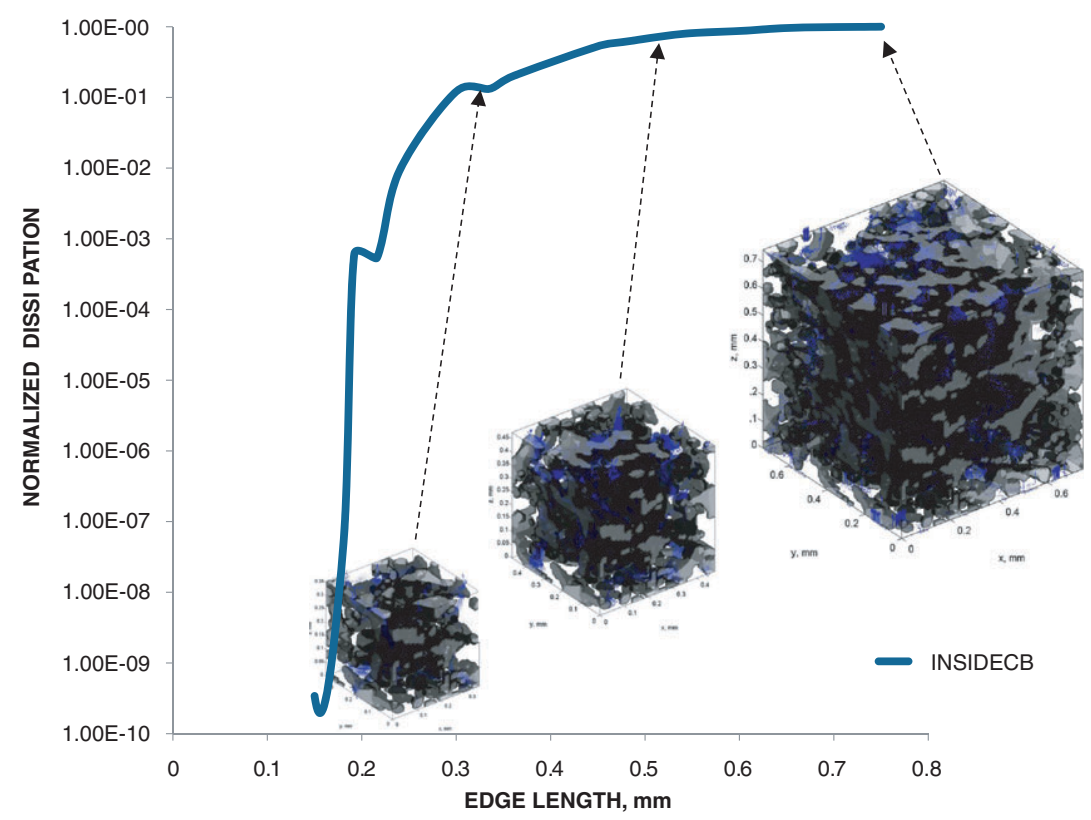

Figure 13. Energy dissipation rate over the edge length of the cubic samples taken inside compaction band. The energy dissipation rate is normalized with respect to the energy dissipation rate of the largest sample with edge length $=0.75 \mathrm{~mm}$.

space region is grown inside the pore space until all connections of the pore network are explored. Figure 12 shows the connected pore space for the $0.54 \times 0.54 \times 0.54 \mathrm{~mm}$ unit cell described above. Using this connected pore space, we calculated an average connected porosity in the compaction band of about $7 \%$. This is basically half of the total porosity and should be contrasted with the $19 \%$ connected porosity in the host rock. One can conclude that the compaction band reduces the available connected porosity considerably relative to the otherwise intact rock.

The next step in our analysis is to determine the minimum appropriate size of unit cells to upscale permeability. Our goal is to estimate permeability along the length of the entire $2.5 \times 2.5 \times 6.00 \mathrm{~mm}$ sample. To do this, as described before, we will use domain decomposition and then use LB/FEM to upscale permeability. In order to establish the minimum cell size, we make use of $D^{\text {micro }}$ as shown in Equation (25). Figure 13 shows the energy dissipation rate as a function of cell size for a typical sample inside the specimen. It can be observed that most fluctuations are eliminated by the time the cell size is greater than $0.4 \mathrm{~mm}$. This result is representative of all other regions in the specimen. This means that any cell size larger than $0.4 \mathrm{~mm}$ in dimensions would be appropriate for continuum representation. We select a convenient cell size of $0.75 \times 0.75 \times 0.75 \mathrm{~mm}$ and therefore decompose the entire sample domain using $3 \times 3 \times 8$ cells.

Decomposing the domain into 72 cells, we can perform multiscale analysis by estimating permeability in each cell using LB and then passing the effective permeability of the subregions into a finite element model with 72 brick elements. Figure 14 shows the entire sample with the average porosity in each cell. It can be seen that the porosity field fluctuates around a mean of $14 \%$. Furthermore, accuracy of the upscaling is monitored by using $D^{\text {macro }}$ as described previously. Hence, the current domain decomposition satisfies the continuum approximation and the accuracy conditions upon scaling.

A macroscopic permeability test is performed along the longitudinal direction of the sample shown in Figure 14. The lateral sides of the specimen are not allowed to pass fluid and the top and bottom faces are prescribed a fluid pressure of 100 and $0 \mathrm{kPa}$, respectively. From this macroscopic permeability calculation, we extract the effective permeability of the sample along the longitudinal direction, $2.5 \times 10^{-13} \mathrm{~m}^{2}$. This is to be contrasted to the effective permeability outside the compaction band, within the host rock, which is $1.4 \times 10^{-12} \mathrm{~m}^{2}$, an order of magnitude 

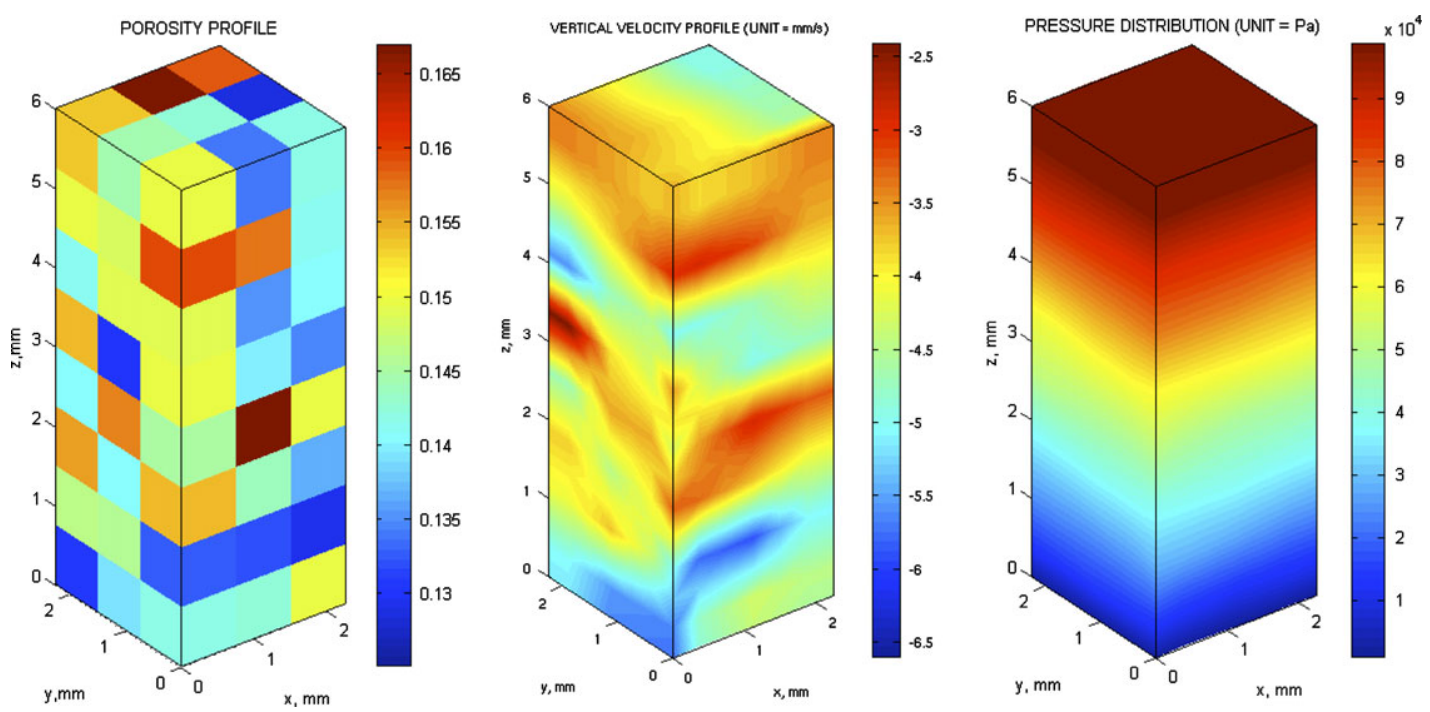

Figure 14. Results of multiscale effective permeability analysis inside compaction band. Color maps represent: (a) porosity; (b) vertical velocity field; and (c) pore pressure.

Table II. Dissipation rates computed via spatial averaging and prescribed values at boundaries.

Volume averaged dissipation rate, $\mathrm{J} / \mathrm{s}$ per $1 \mathrm{~m}^{3}$

difference. These results show a smaller drop in permeability than other studies which report drops between two and three orders of magnitude [17,30,31]. This discrepancy may be due to the fact that the permeability in the literature such as [17,31] are obtained via stochastically reconstructed pore space from 2D SEM images, whereas the calculations presented in this example are conducted on 3D tomographic images directly obtained from X-ray CT. To assess the accuracy of the homogenization procedure, we compute the volume-averaged energy dissipation rate and the energy dissipation rate obtained from boundary conditions. The error of the meso-to-macro homogenization procedure for the compaction band specimen is $1.9 \%$, as shown in Table II. These results show that the LBM/FEM multiscale framework is able to achieve reasonable accuracy, providing that the unit cells are large enough to behave as representative elementary volumes.

It should be noted that all examples shown in this work were conducted in a single-processor machine. The permeability calculations for the compaction band samples are not even feasible without resorting to the multiscale LB/FEM technique proposed in this work.

\section{CONCLUSION}

How does microstructural pore geometry affect the macroscopic fluid transport properties of porous materials? To begin answering this question, we have presented a computational framework that quantifies the interplay between microscale geometrical attributes, directly extracted from tomographic images, and the macroscopic pore fluid properties encapsulated in the effective permeability of the material. Specifically, we have incorporated and expanded a variety of numerical techniques, including semi-implicit level sets, graph theory, and LB coupled with finite element computations exploiting hierarchical multiscale techniques. The numerical techniques proposed are used sequentially with the output from one serving as the input for the next. We have also proposed quantitative criteria to assess the validity of the unit cell size and the accuracy in the multiscale calculations. 
As a result, and as demonstrated by the numerical examples, the computational framework is able to offer tremendous computational advantages over standard approaches without compromising accuracy.

\section{ACKNOWLEDGEMENTS}

This work has been partly funded by the Geosciences Research Program of the U.S. Department of Energy under Grant No. DE-FG02-08ER15980. This support is gratefully acknowledged. We also thank Dr. Nicolas Lenoir for providing the tomographic images of the Aztec sandstone and Dr. David Salac for valuable discussions on the variational level set scheme.

\section{REFERENCES}

1. Lindquist WB, Lee S, Coker DA, Jones KW, Spanne P. Medial axis analysis of void structure in three-dimensional tomographic images of porous media. Journal of Geophysical Research 1996; 101(B4):8297-8310.

2. Dijkstra EW. A note on two problems in connexion with graphs. Numerische Mathematik 1959; 1:269-271.

3. Hill R. Elastic Properties of Reinforced solids: some theoretical principles. Journal of Mechanics and Physics of Solids 1963; 11:357-372.

4. Du X, Ostoja-Starzewski M. On the size of representative volume for Darcy's law in random media. Proceedings of the Royal Society A 2006; 462:2949-2963.

5. Kimmel R, Shaked D, Kiryati N. Skeletonization via Distance Maps and Level Sets. Computer Vision and Image Understanding 1995; 62(3):382-391.

6. Sirjani A, Cross GR. On representation of a shape's skeleton. Pattern Recognition Letters 1991; 12:149-154.

7. Li C, Xu C, Gui C, Fox MD. Level Set Evolution Without Re-initialization: A New Variational Formulation. Proceedings of the 2005 IEEE Computer Society Conference on Computer Vision and Pattern Recognition, IEEE, 2005.

8. Adler PM. Porous Media: Geometry and Transport. Butterworth-Heinemann Series in Chemical Engineering: Stoneham, MA, 1992.

9. Dormieux L, Kondo D, Ulm F.-J. Microporomechanics. Wiley: West Sussex, England.

10. Carman PC. Flow of Gases through Porous Media. Butterworth Scientific Publication: London, 1956.

11. Marcus DA. Graph Theory A Problem Oriented Approach. Mathematical Association of America: Washington, DC, 2008.

12. Lenoir N, Andrade JE, Sun WC, Rudnicki JW. In situ permeability measurements inside compaction bands using X-ray CT and lattice Boltzmann calculations. Proceedings of 3rd International Workshop on X-ray CT for Geomaterials, New Orleans, Louisiana, 2010.

13. Sun WC, Andrade JE. Capturing the effective permeability of field compaction bands using hybrid lattice Boltzmann/finite element simulation. Proceedings of the 9th World Congress of Computational Mechanics, 2010; DOI: $10.1088 / 1757-899 \mathrm{X} / 10 / 012077$.

14. White JA, Borja RI, Fredrich JT. Calculating the effective permeability of sandstone with multiscale lattice Boltzmann/finite element simulations. Acta Geotechnica 2006; 1:195-209.

15. He X, Luo L.-S. Theory of the lattice Boltzmann method: from the Boltzmann equation to the lattice Boltzmann equation. Physical Review E 1997; 56(6):6811-6817.

16. Succi S. The Lattice Boltzmann Equation. Oxford University Press: Oxford, 2001.

17. Keehm Y, Sternlof K, Mukerji T. Computational estimation of compaction band permeability in sandstone. Geosciences Journal 2006; 10(4):409-505.

18. Inamuro O, Yoshino M, Ogino F. A non-slip boundary condition for lattice Boltzmann simulations. Physics of Fluids 1995; 7(12):2928-2930.

19. Bear J. Dynamics of Fluids in Porous Media. American Elsevier: New York, NY, 1972.

20. Berryman JG, Milton GW. Normalization constraint for variational bounds on fluid permeability. Journal of Chemistry Physics 1985; 83(2):754-760.

21. Almon WR, Fullerton LB, Davies DK. Pore space reduction in Cretaceous sandstones through chemical precipitation of clay minerals. Journal of Sedimentary Petrology 1976; 46:89-96.

22. Alvarez D, Abanades JC. Pore-size and shape effects on the recarbonation performance of Calcium Oxide Submitted to Repeated Calcination/Recarbonation Cycles. Energy and Fuels 2005; 19:270-278.

23. Sun WC, Andrade JE, Rudnicki JW. Effect of micro-structural deformation mechanism on the macroscopic effective permeability of compaction bands in Aztec sandstone, in progress.

24. Rudnicki JW. Shear and compaction band formation on an elliptic yield cap. Journal of Geophysical Research 2004; 109:B03402. DOI: 10.1029/2003JB002633.

25. Holcomb D, Rudnicki JW, Issen KA, Sternlof K. Compaction localization in the earth and the laboratory: state of the research and research directions. Acta Geotechnica 2007; 2:1-15.

26. Saeger RB, Scriven LE, Davis HT. Transport processes in periodic porous media. Journal of Fluid Mechanics 1995; 299:1-15. 
W. C. SUN, J. E. ANDRADE AND J. W. RUDNICKI

27. Zick AA, Homsy GM. Stokes flow through periodic arrays of spheres. Journal of Fluid Mechanics 1982; 115:13-26.

28. Crouzeix M, Raviart PA. Conforming and non-conforming finite elements for solving the stationary Stokes equations I. RAIRO Numerical Analysis 1973.

29. Logg A. Automating the finite element method. Archives of Computational Methods in Engineerings 2007; 14(2):93-138.

30. Baxevanis T, Papamichos E, Flornes O, Larsen I. Compaction bands and induced permeability reduction in Tuffeau de Maastricht calcarenite. Acta Geotechnica 2006; 1:123-135.

31. Sternlof KR, Chapin JR, Polland DD, Durlofsky LJ. Permeability effects of deformation band arrays in sandstone. AAPG Bulletin 2004; 88(9):1315-1329. 